\title{
The Nature and Origin of Molecular Knots in Planetary Nebulae
}

\author{
S. E. Eyermann*, A. K. Speck*, M. Meixner ${ }^{*}$, P. R. McCullough ${ }^{\#}$ J. Hora ${ }^{+}$ \\ * Department of Physics and Astronomy; University of Missouri; Columbia, MO 65211 \\ \# Space Telescope Science Institute, 3700 San Martin Drive, Baltimore, MD 21218 \\ ${ }^{+}$Harvard-Smithsonian Center for Astrophysics, 60 Garden Street, MS 65, Cambridge, MA 02138
}

\begin{abstract}
Planetary Nebulae (PNe) are major contributors to the enrichment of the interstellar medium (ISM). Knots and filaments in the ionized gas images of PNe are common, if not ubiquitous. Additionally, it has been shown that molecular gas exists inside dense condensations within the ionized regions. The origins of these clumps are not known, though the suggested formation mechanisms fall into two main scenarios: (1) they form during the AGB phase; (2) they form as a result of the onset of the PN phase as the fast wind ploughs into the slower moving AGB wind. The currently favored model is that the knots are formed by the onset of the PN phase and then sculpted as the ionizing radiation penetrates deeper into the circumstellar envelope. We present preliminary results of a study which compares the morphologies of molecular and ionized gas emission in several $\mathrm{PNe}$, covering a range of ages.
\end{abstract}

\section{INTRODUCTION}

During the AGB phase of stellar evolution in stars with low to intermediate mass, there is heavy mass loss leading to the formation of a dense circumstellar shell of gas and dust. This material is dispersed into the interstellar medium (ISM) during the proto-planetary nebula (PPN) and planetary nebula (PN) phases. As a result, these stars are important contributors to the ISM. The distribution of the gas and dust as it is dispersed has an impact on how it is processed in the ISM.

A basic classical model of a PN has a hot central star with empty space surrounding it where the gas and dust have drifted away with time. Surrounding that is an onion-like layering with the most ionized gas towards the center, and molecular gas at the edges. Dust is distributed all the way through this region. However, if we look at an actual image of a PN, this is not quite what we see. The central zone of the Helix Nebula is filled with emission from ionized helium and is thus not empty space. The clumpy structure in the ionized gas emission is not explained by the previous model. Comparison of the distribution of emission from both ionized and molecular gases in the Helix Nebula shows similar clumps of molecular material in the ionized region, and the locations of the molecular gas clumps and the knots in the ionized gas coincide (Speck et al. 2002).

\section{MOLECULAR KNOTS}

A closer image of the clumps in the Helix Nebula shows knots with bright rounded edges pointing towards the central star and comet-like tails trailing away (O'Dell \& Handron, 1996). This leads to a revised model of a planetary nebula, with no empty central region and with clumps of dust in the ionized region protecting molecular gas from the ionizing radiation. This is shown schematically in Figure 1.

The distribution of the knots in the ionized gas in several PNe was examined by O'Dell et al. (2002). This study showed an evolution in the knots that seemed to correspond to the age of the nebula, with those in older nebulae being smaller and well-formed, and those in younger nebulae being larger and less well sculpted. Speck et al. (2003) showed that one of the nebulae studied (the Ring Nebula) has clumpy $\mathrm{H}_{2}$ emission. Furthermore. the knots in the ionized and molecular gas images were shown to correlate, leading us to expect an evolution in the molecular knots similar to that seen in the knots from the ionized gas images.

\section{$\mathrm{H}_{2}$ Excitation}

In order to observe $\mathrm{H}_{2}$ emission, the gas must be excited. There are two possible methods of excitation. The first is shock excitation, where shocked gas 
regions result from interaction of the fast central star wind with the slowly expanding nebula. The second is

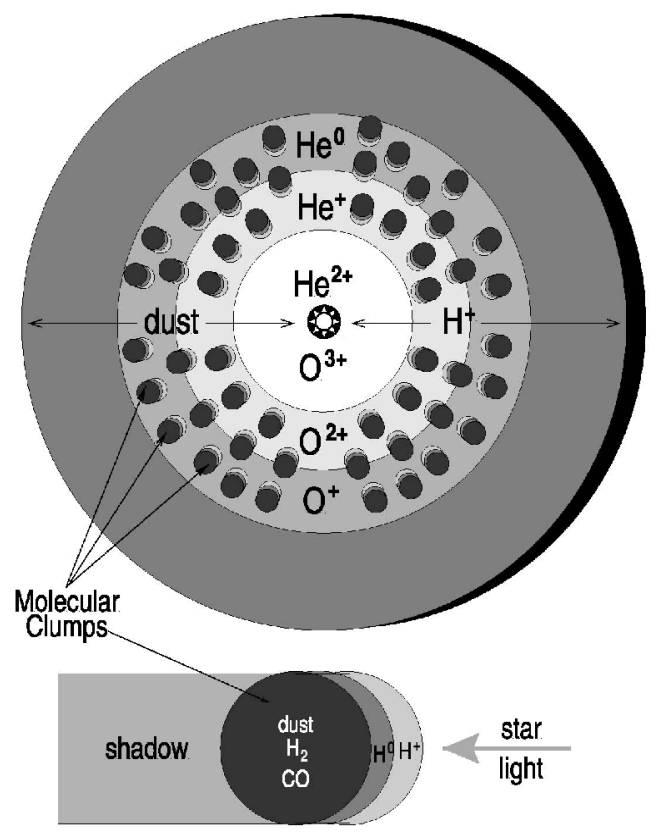

FIGURE 1. This revised model of a planetary nebula accounts for both the pattern of ionized gas seen around the central star and the clumps of molecular material found in the ionized region.

photodissociation regions, or PDRs, where the gas is excited by the far-UV and X-ray radiation of the central star. In the case of such an excitation mechanism, we expect to see a thin layer of ionized gas emission on the surface of the clump facing the central star, with $\mathrm{H}_{2}$ emission behind the ionized gas with respect to the star. The thin bright edge mentioned earlier in the Helix knot images corresponds well to what we would expect from PDR excitation.

In 2002, Huggins, et. al. published work on a single Helix knot. They found that the strongest $\mathrm{H}_{2}$ emission occurs at the face of the globule pointing toward the central star, with little emission directly behind the globule. The observable $\mathrm{H}_{2}$ emission extends out to extremely large distances (up to and beyond 24") and closely follows the ionized gas emission. They concluded that the $\mathrm{H}_{2}$ distribution meet with expectations of $\mathrm{H}_{2}$ excitation in a thin PDR at the surface of the molecular gas and is unlikely to be caused by shocks.

\section{Knot Formation}

In addition to the uncertainty about the excitation of the gas in these molecular knots, there is also uncertainty about when and how they form. They could form before the onset of the PN phase (e.g. Dyson et al. 1989), in which case the material entering the ISM during the AGB phase is already clumpy. Alternatively, they could form as a result of the onset of the PN phase through turbulent instabilities where the fast and slow winds meet (e.g. Capriotti 1971; Vishniac 1994), in which case the material entering the ISM during the AGB phase is relatively smooth. The material will be processed differently in the ISM in each case. By studying the nature of the knots, both in molecular and ionized gas emission, for a large number of $\mathrm{PNe}$, we hope to resolve this issue.

\section{WHAT WE LOOK FOR}

In order to study this problem, we compare the ionized and molecular gas images and determine whether the knots and filaments are spatially coincident with the molecular clumps. So far this has only been shown for the Helix and the Ring Nebulae. With a large enough sample we can determine whether there is a pattern in the sense that the distributions appear to change with the age of the nebula, as has been indicated for the ionized gas by O'Dell et al. (2002). Finally, by studying how the appearance of the knots varies with radial distance from the central star we can determine the role of the ionizing radiation from the central star in the formation and evolution of the knots

To this end, Meixner et. al. (2005) examined molecular knots in the Helix Nebula. The emission from $\mathrm{H}_{2}$ gas was imaged by NICMOS. Figure 2 shows the regions observed.

These new NICMOS images provided a much higher resolution and sensitivity than had been obtained before. Previous $\mathrm{H}_{2}$ images had resolutions between $2^{\prime \prime}$ and $40^{\prime \prime}$, as compared to the $0.01^{\prime \prime}$ resolution obtained in HST optical images. These new images were taken at $0.2^{\prime \prime}$ resolution and show $\mathrm{H}_{2}$ emission where previously none had been detected.

Figure 3 shows an image from position 1 in Figure 2 , with the central star to the top and right of the image. A multitude of knots are visible, with knots on top of other knots, which is something that had not been resolved before. The molecular hydrogen appears in a thin face of emission pointing toward the star like that associated with a PDR.

This study concluded that there are up to 162 knots/arcmin ${ }^{2}$ in the denser regions toward the center and down to $18 \mathrm{knots} / \mathrm{arcmin}^{2}$ in the outer regions. This gives us an estimate of about 23,000 total knots in the Helix, which is about 6.5 times larger than previous estimates. Furthermore, the structure and evolution of the knots changes with radial distance from the central star, suggesting that the ionizing radiation plays a 
major role in determining the structure, and possibly the origin, of these knots.

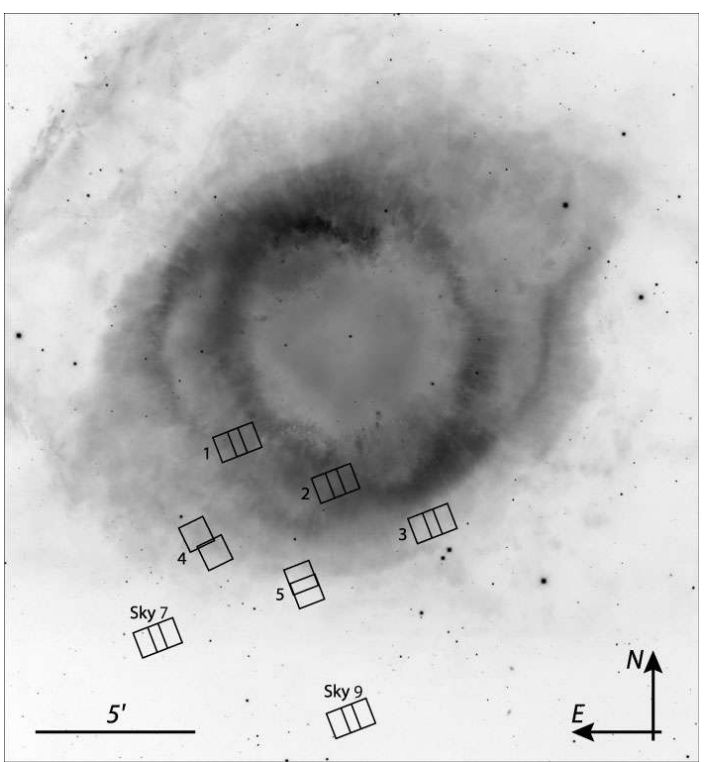

FIGURE 2. This negative picture of the Helix from O'Dell et al. (2004) shows the optical structure on a large scale. The NICMOS filed positions are overlaid as black squares.

\section{FUTURE WORK}

Recent Spitzer observations of the molecular hydrogen in two PNe (the Ring and the Dumbbell nebulae; PI: Hora) show the need for higher resolution and higher sensitivity $\mathrm{H}_{2}$ images. In the Ring Nebula, the outer petal structure is seen more clearly than in previous $\mathrm{H}_{2}$ observations, and clearly follows the distribution of ionized gas (Balick et al. 1992). Detailed comparisons are now required. Expanding our studies to these outer petals will improve our understanding of the radial distribution of the $\mathrm{H}_{2}$ in PNe. The Dumbbell has been observed with WFPC2, and thus detailed comparisons of the ionized and molecular gas distributions in this object are also possible.

Further studies of the ionized and molecular gas in a range of $\mathrm{PNe}$ can also be achieved using both archival and new HST observations.

\section{ACKNOWLEDGMENTS}

This work was supported by several funding agencies: SE and AS were supported by HST-AR10686, and by the Research Council of University of Missouri - Columbia. MM and PM were supported in part by HST-GO-01041 and by the internal STScI funds, DDRFD0001.82319. Support for Program numbers HST-AR-10686 and HST-GO-01041 was provided by NASA through a grant from the Space Telescope Science Institute, which is operated by the

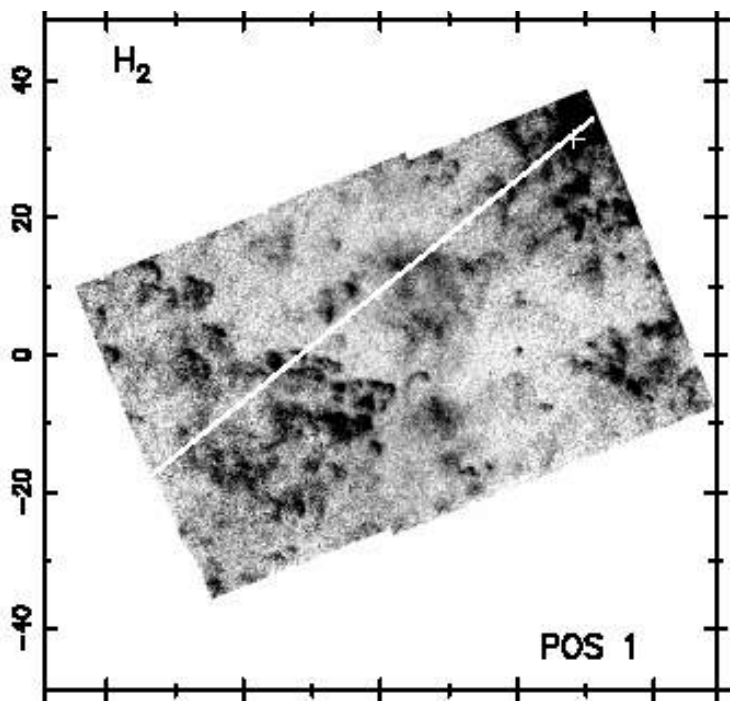

FIGURE 3. High resolution NICMOS image in $\mathrm{H}_{2} 2.12 \mu \mathrm{m}$ of a portion of the Helix Nebula.

Association of Universities for Research in Astronomy, Incorporated, under NASA contract NAS5-26555.

\section{REFERENCES}

Balick, B., et al. 1992, ApJ, 392, 582

Capriotti, E. 1971, ApJ, 166, 563

Dyson, J.E., et al. 1989, MNRAS, 241, 625.

Huggins, P., et al. 2002, ApJ, 346, 201

Meixner, M., McCullough, P. R., Hartman, J., et al. 2005, $\mathrm{AJ}$, in press

O’Dell, C. R., \& Handron, K.D. 1996, AJ, 111, 1630

O'Dell, C. R., Balic, B., Hajian, A. R., et al. 2002, AJ, 123, 3329

O’Dell, C. R., McCullough, P. R, Meixner, M. 2004, AJ, 128,2339

Speck, A. K., Meixner, M., Fong, D., et al. 2002, AJ, 123, 346

Speck, A. K., Meixner, M., Jacoby, G. H., et al. 2003, PASP, 115,170

Vishniac, E.J. 1994, ApJ, 428, 186. 